\title{
OPEN A stable spin-structure found in a 3-body system with spin-3 cold atoms and its role in $\mathrm{N}$-body condensates
}

\begin{abstract}
Y. M. Liu ${ }^{1,3}$, Y. Z. He $\mathrm{He}^{2}$ \& C. G. Bao ${ }^{2 \bowtie}$
We have found a stable spin-structure of $\mathrm{N}=3$ system in which three spin-3 atoms are trapped and coupled to total spin $\mathrm{S}=3$. We have proved that a pair of this structure is nearly an exact solution for $\mathrm{N}=6$ systems in a very broad district in the parameter-space. Comparing with the well-known singlet pairs, this pair is a more promising candidate to serve as a building block for large $\mathrm{N}$ systems with spin-3 atoms. This is because the spin-structure of the latter can be modified depending on the interactions to reduce the energy while the singlet pairs can not. In fact, we have proved that, for a specific set of strengths (a point in the parameter-space) the product state based on this pair is an exact solution of the $\mathrm{N}$-body Hamiltonian. Thus, in the neighborhood of this point, the product state will appear as an approximate solution. However, how broad this neighborhood would be remains to be clarified.
\end{abstract}

It is well known that the study of the Bose-Einstein condensates as an artificial matter is important in the academic sense and for practical applications. In particular, since the realization of optical trapping ${ }^{1-9}$, the study of the spinor condensates has become a hot topic. When the temperature is very low, the spatial degrees of freedom are nearly frozen and the spin-degrees of freedom play essential roles. For this case, the understanding of the spin-structures is crucial. Various structures (phases) have already been found. For condensates with spin-1 atoms, the ground state (g.s.) may have the ferro-phase ( $f$-phase, where all the spins are aligned along the same direction) and polar-phase ( $p$-phase, where all the spins are two-by-two couples to zero and form the singletpairs). For spin- 2 condensates, the $f$-phase, $p$-phase, together with the cyclic-phase ( $c$-phase, where the g.s. is nearly a product-state of the triplets, in each triplet the three spin-2 atoms are coupled to zero) are found ${ }^{8,10-17}$. There are also studies for spin-3 condensates, where the structures appear to be complicated ${ }^{18-24}$.

Due to the progress in technology, it is possible to put only a few atoms in a trap. In the theoretical aspect, instead of using mean-field theory, exact solutions for few-body systems can be obtained. The knowledge extracted from few-body systems would be a complement to those from many-body theories. Furthermore, these few-body cold systems might be more suitable for realistic applications because they can be more precisely controlled.

The present paper is dedicated to the study of spin-3 cold atoms. The main purpose is to find out some stable constituents from few-body systems and to evaluate their potential for serving as a building block for large $N$ systems.

\section{Exact solutions of the 3-body Schrödinger equation}

Let three spin-3 atoms (say, $\mathrm{Cr}, \mathrm{Mo}, \mathrm{Sn}, \mathrm{Pu}$ ) be confined in an optical trap. It is assumed that the temperature is so low and the binding is so strong that all the particles have condensed to the same spatial state $\phi(\mathbf{r})$ with zero orbital angular momentum. The state $\phi(\mathbf{r})$ is most favorable for binding, the excitation of this state is not considered. When all the spatial degrees of freedom have been frozen, only the spin-degrees of freedom are necessary to be considered. Then, the Hamiltonian can be written as

\footnotetext{
${ }^{1}$ Department of physics, Shaoguan University, Shaoguan 510205, People's Republic of China. ${ }^{2}$ School of Physics, Sun Yat-Sen University, Guangzhou 510275, People's Republic of China. ${ }^{3}$ State Key Laboratory of Theoretical Physics, Institute of Theoretical Physics, Chinese Academy of Sciences, Beijing 100190, People's Republic of China. ${ }^{\varpi}$ email: stsbcg@mail.sysu.edu.cn
} 


$$
\begin{gathered}
H=\sum_{i<j} V_{i j}, \\
V_{i j}=\sum_{\lambda} g_{\lambda} P_{\lambda}^{i j},
\end{gathered}
$$

where $i(j)$ denotes the particle 1 to partical 3. $\lambda=0,2,4$, and 6 is the coupled spin of two particles, $P_{\lambda}^{i j}$ is the projector to the $\lambda$-channel. $g_{\lambda}$ is the weighted strength where a factor $\int \phi^{4} d \mathbf{r}$ is contained. This factor embodies the effect of the spatial wave function $\phi$ on the spin-structures. Since $\phi$ has orbital angular momentum zero, the orbital-spin coupling is suppressed and therefore is neglected. The dipole-dipole $(d-d)$ coupling between a pair of atoms is relatively weak (for ${ }^{52} \mathrm{Cr}$ as an example, the strength of the $d$ - $d$ coupling $c_{d d}=0.004 g_{6}$ ), therefore it is also neglected. In fact, the calculation in ${ }^{19}$ demonstrates that the g.s. of ${ }^{52} \mathrm{Cr}$ does not seem to depend on the $d-d$ coupling.

An important feature of $H$ is the conservation of the total spin $S$. Due to symmetry constraint, $S$ is allowed to be equal to $1,3,4$ to 7 , and 9 . The corresponding total spin-states $\psi_{S}$ are studied and given below.

The case $S \neq 3$. In this case the multiplicity of $\psi_{S}$ is one. It can be written as $\psi_{S}=\mathfrak{P}\left((\chi \chi)_{\lambda} \chi\right)_{S}$, where $\chi$ denotes the spin-state of a spin-3 atom, the first two spin-states are coupled to $\lambda$, then the three are coupled to $S$. $\mathfrak{P}$ is the operator for symmetrization and normalization. $\lambda$ is an even number and $|3-S| \leq \lambda \leq 3+S$. Since the multiplicity of $\psi_{S}$ is one, $\lambda$ can be arbitrary chosen (i.e., different choices of $\lambda$ lead to the same $\bar{\psi}_{S}$ ).

By re-coupling the three spins, $\psi_{S}$ can be rewritten as

$$
\psi_{S}=\sum_{\eta} C_{\lambda \eta}^{S}\left((\chi(1) \chi(2))_{\eta} \chi(3)\right)_{S M},
$$

where $\eta$ should be even,

$$
C_{\lambda \eta}^{S}=\left(\delta_{\lambda \eta}-2(-1)^{S} \sqrt{(2 \lambda+1)(2 \eta+1)} W(33 S 3 ; \lambda \eta)\right) / \mathfrak{N}
$$

where the $\mathrm{W}$ coefficient of Racah has been introduced, the constant $\mathfrak{N}$ is introduced to assure $\sum_{\eta}\left(C_{\lambda \eta}^{S}\right)^{2}=1$. In Eq. (3) particles 1 and 2 have been extracted. Note that, due to the symmetry, the labels 1,2 , and 3 at the right side of Eq. (3) can be replaced by any other permutations of the three numbers. Making use of this feature and the multiplicity of $\psi_{S}$ we can prove

$$
\begin{gathered}
H \psi_{S}=E_{S} \psi_{S}, \\
E_{S}=3 \sum_{\eta} g_{\eta}\left(C_{\lambda \eta}^{S}\right)^{2} .
\end{gathered}
$$

Note that $\psi_{S}$ as an eigen-state of $H$ does not depend on $\left\{g_{\eta}\right\}$ but simply on symmetry. Since the choice of $\lambda$ is irrelevant, $C_{\lambda \eta}^{S}$ can be rewritten as $C_{\eta}^{S}$ in short.

Inserting Eq. (3) into the equation $\left\langle\psi_{S} \mid \psi_{S}\right\rangle=1$, we can deduce that the probability of an atom lying at the $\mu$ -component $(-3 \leq \mu \leq 3)$ is

$$
P_{\mu}^{S M}=\sum_{\eta}\left(C_{\eta, M-\mu ; 3, \mu}^{S M} C_{\eta}^{S}\right)^{2},
$$

The probability that two atoms are lying at $\mu$ and $\nu$, respectively, are

$$
P_{\mu \nu}^{S M}=\sum_{\eta}\left(C_{\eta, M-\mu ; 3, \mu}^{S M} C_{3, \nu ; 3, M-\mu-\nu}^{\eta, M-\mu} C_{\eta}^{S}\right)^{2} .
$$

Furthermore, from Eq. (3), $\left(C_{\eta}^{S}\right)^{2}$ is the probability of a pair of particles being coupled to $\eta$, This explains the origin of $E_{S}$ as given in Eq. (6).

The case $S=3$. When $S=3$ the multiplicity is two. The two spin-eigen-states are denoted as $\psi_{3 k}(k=1$ for the lower and 2 for the higher). They can be expanded as

$$
\psi_{3 k}=\sum_{\eta} C_{\eta}^{3 k}\left((\chi(1) \chi(2))_{\eta} \chi(3)\right)_{3}
$$

where $C_{\eta}^{3 k}$ depends on $\left\{g_{\eta}\right\} . \psi_{3 k}$ can also be analytically obtained as shown in Suppl. Appendix 1. The associated eigen-energy $E_{3 k}=3 \sum_{\eta} g_{\eta}\left(C_{\eta}^{3 k}\right)^{2}$.

Up to now, with the freezing of the spatial degrees of freedom, all the eigen-states and eigen energies of the $N=3$ system have been found. each specifies a kind of spin-structure. It is evident that the multiplicity is very important to the spin-structures of few-body systems. When the multiplicity is one, the structure is irrelevant to dynamics but completely determined by symmetry. Whereas for those spin-states with multiplicity $\geq 2$, they can be modified by adjusting the strengths of interaction. These states are noticeable. 

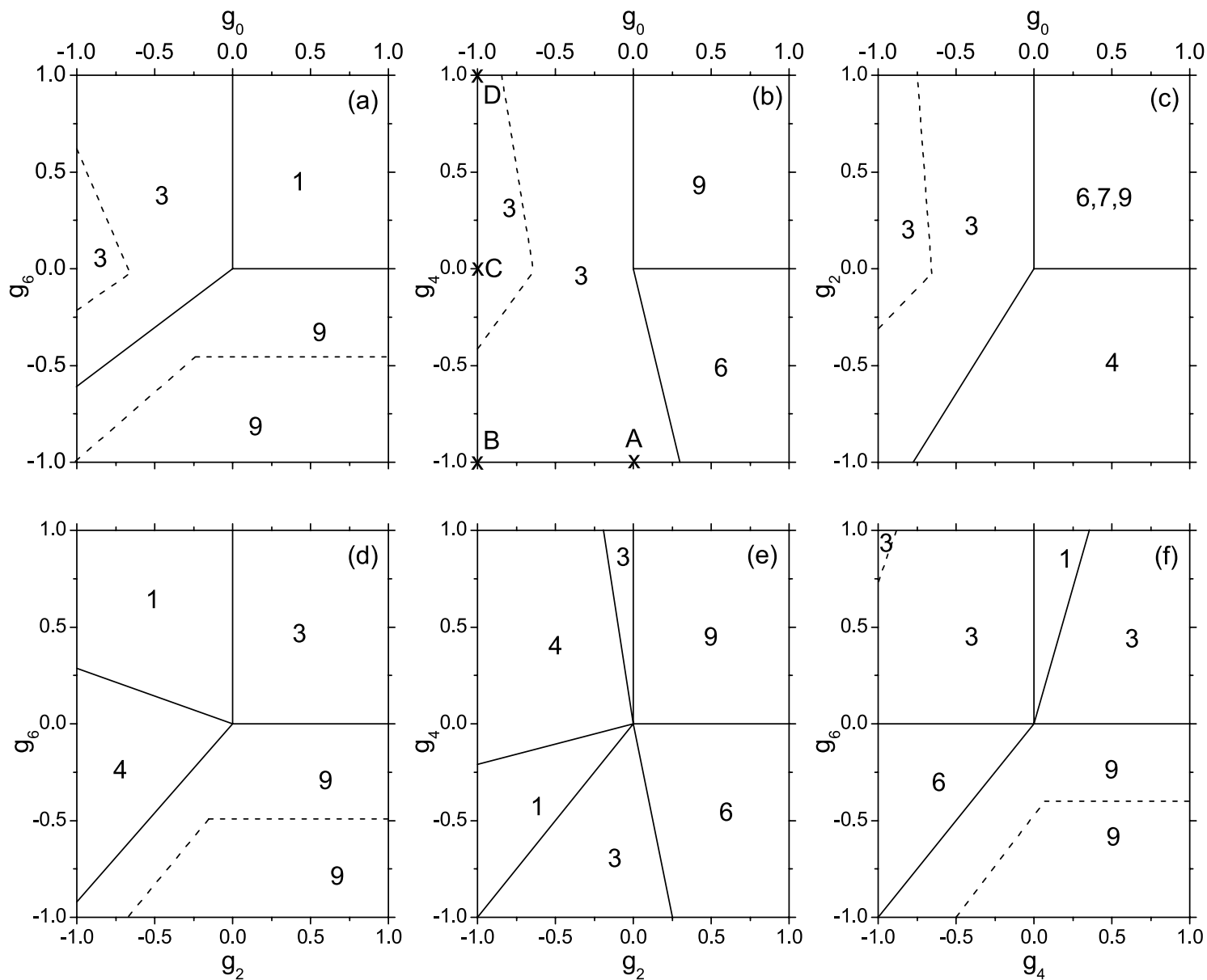

Figure 1. The phase-diagram of a 3-body trapped system with spin-3 cold atoms. In each panel two $g_{\eta}$ are chosen to serve as the ordinate and abscissa. The values of the other two $g_{\eta}$ are given at zero. The phase of the g.s. is specified by the total spin $S$ marked inside the related zone. The district bound by the dotted lines has $E_{\text {gap }} \geq 0.8$.

\section{Phase-diagrams for $\boldsymbol{N}=\mathbf{3}$ systems}

We will neglect the higher state $\psi_{3,2}$, then $\psi_{3,1}$ is rewritten as $\psi_{3}$. Among the seven $\psi_{S}(S=1$ to 9 , except 2 and 8 ), the one having the lowest energy is the g.s. The phase of the g.s. can be specified by $S$. The phase-diagram is plotted in Fig. 1 where the variation of the phase against $\left\{g_{\eta}\right\}$ is shown. For any sets of $\left\{g_{\eta}\right\}$, we find out the two, say, $g_{a}$ and $g_{b}$, having the smallest $\left|g_{a}-g_{b}\right|$. Then, as an approximation, $g_{a}=g_{b}=\frac{g_{a}+g_{b}}{2}$ is assumed. Note that: (i) If all the $g_{\eta}$ are shifted by a common value, then the total energy will shift accordingly but the spin-structures will remain unchanged. (ii) If the unit of energy is changed, the spin-structures will remain unchanged. Thus, we adopt a shift so that $\frac{g_{a}+g_{b}}{2}$ is shifted to zero, and we adopt a new unit so that the scope $-1 \rightarrow 1$ is sufficient. Then, the 4 -dimensional complicated phase-diagram can be replaced by six 2 -dimensional diagrams. They are sufficient to reveal the qualitative features. In order to understand the stability of the g.s., the energy gap $E_{\text {gap }}$ (the energy difference between the first excited state and the g.s.) has been calculated. The districts with $E_{g a p} \geq 0.8$ are marked, in which the g.s. is relatively more stable (the choice 0.8 is quite arbitrary, it is so chosen that a smaller part of the whole zone appears as a relatively more stable zone.).

The feature of a spin-structure is inherent in the coefficients $\left\{C_{\eta}^{S}\right\}$ which arises from symmetry constraint. Their squares are listed in Table 1 . It is Noted that these coefficients does not depend on $\left\{g_{\eta}\right\}$ except those for $S=3$ states. In the latter case four sets of $\left\{g_{\eta}\right\}$ are chosen as examples, they are associated with the points A to D marked in Fig. 1b.

From this table we see that

- Since $\psi_{9}$ has $C_{6}^{9}=1$, the contribution from $g_{6}$ is maximized. Therefore, when $g_{6}$ is the smallest (most negative), $S=9$ is the best choice. This leads to the $f$-phase. The appearance of this phase in large $N$ systems has been well known.

- When $g_{0}$ is the smallest, due to all $C_{0}^{S}=0$ if $S \neq 3$, the $S=3$ is the best choice.

- When $g_{4}$ and $g_{6}$ both are smaller (this happens in the up-right corner of Fig. 1c and the down-left corner of Fig. 1f), the states with a larger $\left(C_{4}^{S}\right)^{2}$ and $\left(C_{6}^{S}\right)^{2}$ will be lower. Therefore the candidates of the g.s. are those with $S=6,7$, and 9. For them and from Eq. (6), we have $E_{S}=g_{6}+\left(C_{4}^{S}\right)^{2}\left(g_{4}-g_{6}\right)$, where $\left(C_{4}^{S}\right)^{2}=0.727,0.515$, and 0 , respectively, for $S=6,7$, and 9 . Therefore, when $g_{4}<g_{6}$, the best choice is $S=6$ because $\left(C_{4}^{6}\right)^{2}$ is the 


\begin{tabular}{|l|l|l|l|l|}
\hline $\boldsymbol{S}$ & $\left(\boldsymbol{C}_{\mathbf{0}}^{\boldsymbol{S}}\right)^{\mathbf{2}}$ & $\left(\boldsymbol{C}_{\mathbf{2}}^{\boldsymbol{S}}\right)^{\mathbf{2}}$ & $\left(\boldsymbol{C}_{4}^{\boldsymbol{S}}\right)^{\mathbf{2}}$ & $\left(\boldsymbol{C}_{\mathbf{6}}^{\boldsymbol{S}}\right)^{\mathbf{2}}$ \\
\hline 1 & 0 & 0.524 & 0.476 & 0 \\
\hline 3A & 0.108 & 0.136 & 0.753 & 0.003 \\
\hline 3B & 0.234 & 0.031 & 0.687 & 0.049 \\
\hline 3C & 0.429 & 0.106 & 0.190 & 0.275 \\
\hline 3D & 0.380 & 0.263 & 0.025 & 0.331 \\
\hline 4 & 0 & 0.611 & 0.061 & 0.328 \\
\hline 5 & 0 & 0.413 & 0.234 & 0.353 \\
\hline 6 & 0 & 0 & 0.727 & 0.273 \\
\hline 7 & 0 & 0 & 0.515 & 0.485 \\
\hline 9 & 0 & 0 & 0 & 1 \\
\hline
\end{tabular}

Table 1. The squares of the coefficients $\left(C_{\eta}^{S}\right)^{2}$ for the eigen-spin-states of the 3-body system. $3 \mathrm{~A}$ to $3 \mathrm{D}$ are for the $S=3$ states with the parameters given at the points A to D marked in Fig. $1 \mathrm{~b}$.

largest. Whereas when $g_{4}>g_{6}$, the best choice is $S=9$ because $\left(C_{4}^{9}\right)^{2}=0$. In Fig. $1 \mathrm{f}$ the boundary separating the $S=6$ and 9 zones has $E_{6}-E_{9}=0$. This leads to $g_{4}-g_{6}=0$. In fact, once $g_{4}=g_{6}$, the three states with $S=6,7$, and 9 are degenerate as shown in Fig. 1c.

- When $g_{2}$ and $g_{6}$ are smaller (the up-right corner of Fig. $1 \mathrm{~b}$ and the down-left corner of Fig. 1d), the unfavorable contribution from $g_{0}$ and $g_{4}$ would be minimized in $\psi_{4}$ and $\psi_{9}$ because $\left(C_{0}^{S}\right)^{2}$ and $\left(C_{4}^{S}\right)^{2}$ are zero or much smaller if $S=4$ and 9. In Fig. 1b, the up-right corner has $E_{4}-E_{9}=0.061 g_{4}>0$. Thus, the zone has $S=9$. In Fig.1d, the down-left corner has $E_{4}-E_{9}=0.611 g_{2}-0.672 g_{6}$. Therefore, the zone will have $S=4(9)$ if $g_{2}<(>) 1.1 g_{6}$. The boundary separating the $\mathrm{S}=4$ and 9 zones has $g_{2}=1.1 g_{6}$.

Similarly, all the boundaries can be analytically explained. It is reminded that all the spin-structures with $S \neq 3$ are fixed by symmetry constraint. However, $\psi_{3}$ will change against $\left\{g_{\eta}\right\}$. For examples, when the point A in Fig. $1 \mathrm{~b}$ is shifted to B, the g.s. is changed from $\psi_{3 A}$ to $\psi_{3 B}$ (refer to the second and third rows of Table 1 ). The shift implies a decrease of $g_{0}$ and accordingly an increase of $\left(C_{0}^{S}\right)^{2}$. In this way, the g.s. energy is reduced. Similarly, the shift $\mathrm{B} \rightarrow \mathrm{C} \rightarrow \mathrm{D}$ implies an increase of $g_{4}$ and accordingly a decrease in $\left(C_{4}^{S}\right)^{2}$, etc..

For ${ }^{52} \mathrm{Cr}$, when the strength of $g_{6}$ is used as an energy unit, then $g_{6}=1, g_{4}=0.517, g_{2}=-0.063$. By an exact numerical calculation on this $N=3$ system, we found a critical value $g_{\text {crit }}=-0.3$. When $g_{0}<g_{\text {grit }}$ the g.s. has $S=3$, whereas when $g_{0}>g_{\text {grit }}, S=1$. This information would be helpful for identifying $g_{0}$. This realistic case is qualitatively similar to the upper part of Fig. 1a. Where, when $g_{0}$ increases from $-1 \rightarrow 1$ along the upper boundary, $S$ transits from $3 \rightarrow 1$ at the critical value $g_{\text {crit }}=0$.

We found that the more stable districts (bound by the dot line) either have $S=9$ or $S=3$. In the former all the spins are lying along the same direction. In the latter the spin-structure depends on $\left\{g_{\eta}\right\}$. We found from Fig. 1a-c that once $g_{2}=g_{4}=g_{6}=0$ and $g_{0}=-1, E_{\text {gap }}$ of $\psi_{3}$ will arrive at its maximum 1.2857 (say, at the point $\mathrm{C}$ of Fig. 1b). At the maximum the probabilities extracted from $\psi_{3}$ (refer to Eqs. (7) and 8)) are $P_{3}^{3,3}=0.481$, $P_{-3}^{3,3}=0.148$, while all the other $P_{\mu}^{3,3}=0.074$. It implies that, when $M=S$ is chosen and therefore $S$ is essentially lying along the $Z$-axis, the spins are mostly lying along the $\pm Z$-axis. When one spin is given at $\mu=3$, we have $P_{3, v}^{3,3} / P_{3}^{3,3}=0.308$ (if $v= \pm 3$ ) or 0.077 (if $v \neq \pm 3$ ). This leads to an intuitive picture, namely, two spins are mostly lying along $S$ while the third lying reversely. It is interesting to ask whether this relatively more stable structure $\psi_{3}$, in addition to $\psi_{9}$, would play a role as a building block in large $N$ systems. To reply, we first go to $N=6$ system.

\section{Phase-diagrams for $N=6$ systems}

When $N=6$, we do not have analytical solutions. Instead, the solutions are obtained via a diagonalization of the Hamiltonian against the Fock-states as basis-states. The resultant phase-diagrams are given in Fig. 2.

We found that the zones with $S=18$ in Fig. 2 and those with $S=9$ in Fig. 1 overlap nearly. It implies that the $f$-phase would emerge in both systems under similar condition of $\left\{g_{\eta}\right\}$, i.e., $g_{6}$ alone is the smallest one (most negative), or $g_{6}$ and $g_{x}(x=0,2$, or 4$)$ are both smaller. In the latter case there is a competition, say, in the down-left corner of Fig. $1 \mathrm{~d}, g_{6}<g_{2} / 1$.1 is required to assure the $f$-phase. while in Fig. $2 \mathrm{~d}$ a similar condition is found. Therefore, it is expected that the $f$-phase would emerge in large $N$ systems under similar conditions. But this remains to be checked.

Let the $S=0$ exact eigen-states be denoted as $\Psi_{0}$, its multiplicity is 3 . Thus, $\Psi_{0}$ has three kinds of spin-structures. It turns out that the zones with $S=0$ in Fig. 2 are larger but include those with $S=3$ in Fig. 1 . It implies that at least a kind of $\Psi_{0}$ might contain $\psi_{3}$ as a constituent. To clarify, we calculate the overlap $\left\langle\Psi_{0} \mid \mathfrak{P}\left(\psi_{3} \psi_{3}\right)_{0}\right\rangle$ (where the two $\psi_{3}$ states are coupled to zero) as shown in Fig. 3. Let $(\chi \chi)_{0}$ denote a singlet-pair (two atoms are coupled to zero). The overlap $\left\langle\Psi_{0} \mid \mathfrak{P}(\chi \chi)_{0}^{3}\right\rangle$ has also been given in Fig. 3 for a comparison.

Figure 3 demonstrates that, in a rather broad scope of the parameters, the overlap $\left\langle\Psi_{0} \mid \mathfrak{P}\left(\psi_{3} \psi_{3}\right)_{0}\right\rangle$ is $\geq 0.995$. In this case the g.s. can be nearly exactly described by $\mathfrak{P}\left(\psi_{3} \psi_{3}\right)_{0}$. Whereas $\left\langle\Psi_{0} \mid \mathfrak{P}(\chi \chi)_{0}^{3}\right\rangle$ is in general not close to 1 except in a very narrow district around the point with $g_{0}=-1$ and $g_{2}=g_{4}=g_{6}=0$. At the point both overlaps are equal to 1 . In this particular case both $\mathfrak{P}\left(\psi_{3} \psi_{3}\right)_{0}$ and $\mathfrak{P}(\chi \chi)_{0}^{3}$ are identical to the exact solution. A distinguished feature of $\mathfrak{P}\left(\psi_{3} \psi_{3}\right)_{0}$ is its flexibility against the strengths, i.e., it can be adjusted to reduce the 

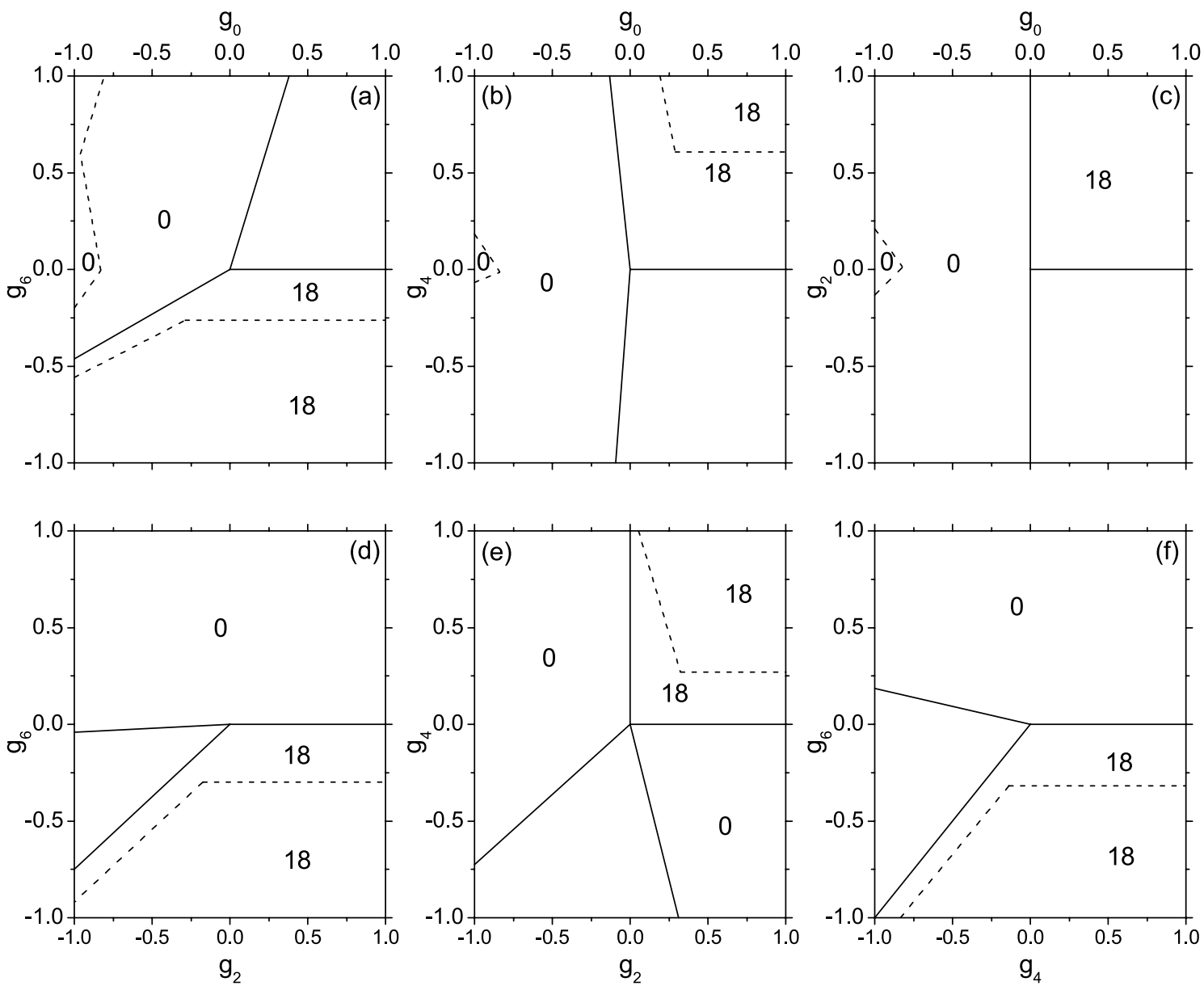

Figure 2. The phase diagrams for $N=6$ systems (refer to Fig. 1). Only the zones with $S=0$ and 18 are marked.

energy while $\mathfrak{P}(\chi \chi)_{0}^{3}$ cannot. This explains why the former surpasses the latter. Thus the former would be a better candidate of building block.

\section{Large $\boldsymbol{N}$ systems and final remarks}

At the point in the parameter-space with $g_{0}<g_{2}=g_{4}=g_{6}$ (this set is equivalent to $g_{2}=g_{4}=g_{6}=0$ and $g_{0}$ is negative), we can prove $\mathfrak{P}\left(\psi_{3} \psi_{3}\right)_{0}^{K}=\mathfrak{P}(\chi \chi)_{0}^{3 K}$ (if $\left.N=6 K\right)$ or $\mathfrak{P}\left(\psi_{3} \psi_{3}\right)_{0}^{K} \psi_{3}=\mathfrak{P}(\chi \chi)_{0}^{3 K}+1$ (if $\left.N=6 K+3\right)$. In particular, all of them are exact solutions of the Hamiltonian. The proof is given in Suppl. Appendix 2. This fact implies that these product states of the building blocks would be good approximate solutions for the g.s. at least in the neighboring district around the point. Due to the flexibility of the building block $\left(\psi_{3} \psi_{3}\right)_{0}$, the product state based on $\left(\psi_{3} \psi_{3}\right)_{0}$ is expected to be valid in a much broader district than that based on $(\chi \chi)_{0}$ as shown in Fig. 3 . Thus the pair $\left(\psi_{3} \psi_{3}\right)_{0}$ is superior than the singlet pair to serve as a building block for large $N$ system.

In conclusion, we have studied the features of the spin-states of the $N=3$ system. We found that, in addition to the $f$-phase, where all the three spins are lying along the same direction, $\psi_{3}$ is also very stable, where two spins are essentially lying along the same direction while the third lying reversely. In particular, the $\left(\psi_{3} \psi_{3}\right)_{0}$ pair has been proved to be a nearly exact eigen-states for $N=6$ systems in a rather broad sub-space in the parameter space. The $\left(\psi_{3} \psi_{3}\right)_{0}$ pair is a promising candidate, superior to the singlet pair $(\chi \chi)_{0}$, for serving as a building block for $N$-body systems. Although the product state based on $\left(\psi_{3} \psi_{3}\right)_{0}$ has been proved to be an exact solution at the point with $g_{0}<g_{2}=g_{4}=g_{6}$, how broad is the district around the point in which the product state could be considered as a good approximation remains to be clarified.

In this paper a stable sub-structure has been extracted from $N=3$ system. It is likely that stable spin structures might also exist in $N \geq 4$ systems. For an example, the $S=4$ state of $N=4$ system with spin-3 atoms has multiplicity 3 , thus this state has a better flexibility (the ability to modify its structure to reduce the energy). Therefore, it might serve as a building block for large $N$ systems when the strengths are given in a specific region. Nonetheless, this is only a presumption. The role of the stable sub-structures from $N \geq 4$ systems remains to be clarified. 

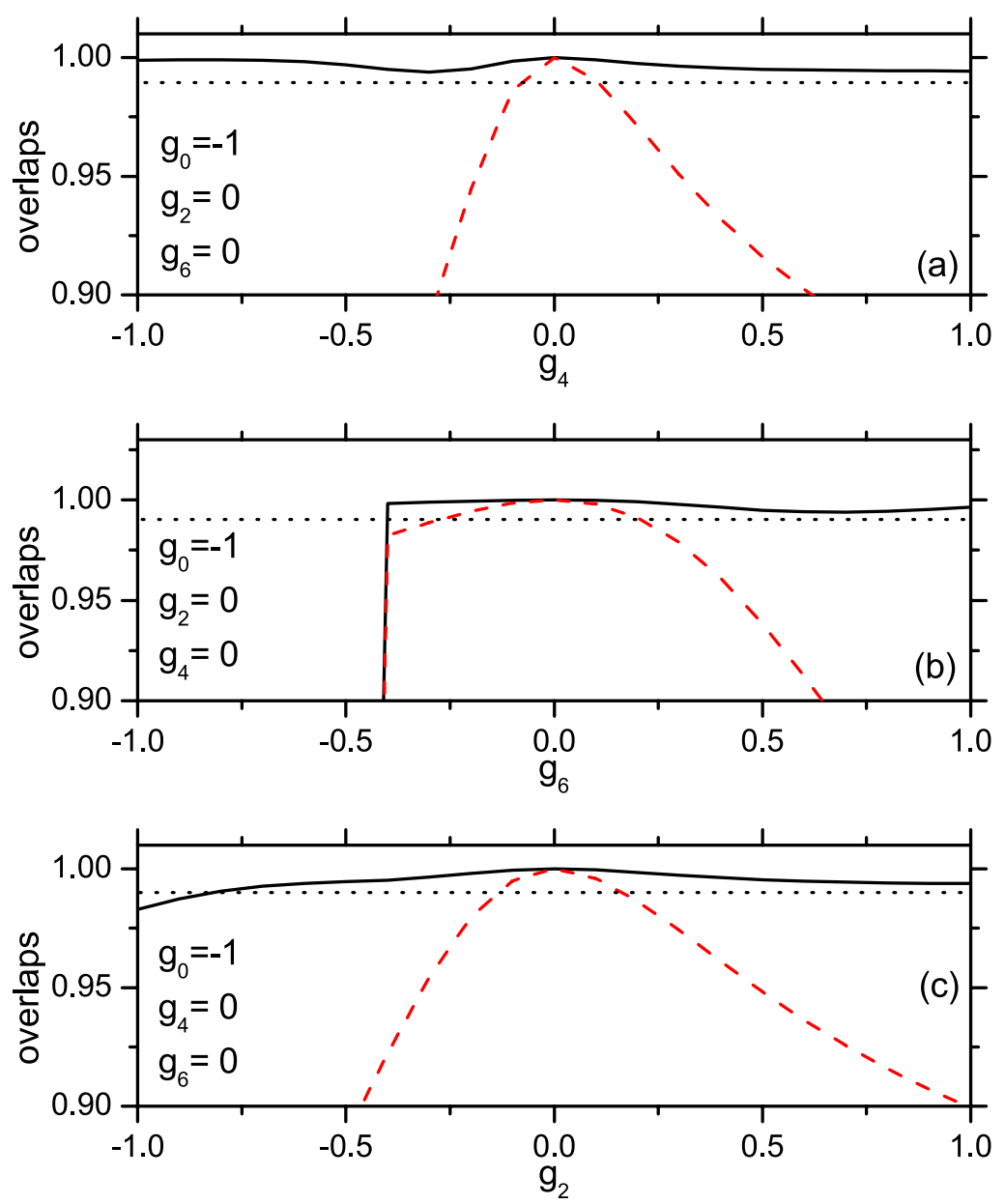

Figure 3. The overlaps $\left\langle\Psi_{0} \mid \mathfrak{P}\left(\psi_{3} \psi_{3}\right)_{0}\right\rangle$ (solid line) and $\left\langle\Psi_{0} \mid \mathfrak{P}(\chi \chi)_{0}^{3}\right\rangle$ (dashed line) against $g_{4}, g_{6}$, or $g_{2}$. In Fig. $3 \mathrm{a}-\mathrm{c}$, The varying strength is moving up along the left boundary of Fig.2b,a,c, respectively. The dotted horizontal line marks the value 0.99 .

Received: 6 May 2020; Accepted: 16 December 2020

Published online: 19 January 2021

\section{References}

1. Stenger, J. et al. Spin domains in ground-state Bose-Einstein condensates. Nature 396, 345 (1998).

2. Stamper-Kurn, D. M. et al. Optical confinement of a Bose-Einstein condensate. Phys. Rev. Lett. 80, 2027 (1998).

3. Ho, T. L. Spinor Bose condensates in optical traps. Phys. Rev. Lett. 81, 742 (1998).

4. Ohmi, T. \& Machida, K. Bose-Einstein condensation with internal degrees of freedom in alkali atom gases. J. Phys. Soc. Jpn. 67, 1822 (1998)

5. Law, C. K., Pu, H. \& Bigelow, N. P. Quantum spins mixing in spinor Bose-Einstein condensates. Phys. Rev. Lett. 81, 5257 (1998).

6. Goldstein, E.V. \& Meystre, P. Quantum theory of atomic four-wave mixing in Bose-Einstein condensates. Phys. Rev. A 59, 3896 (1999).

7. Ho, T. L. \& Yip, S. K. Fragmented and single condensate ground states of spin-1 Bose gas. Phys. Rev. Lett. 84, 4031 (2000).

8. Koashi, M. \& Ueda, M. Exact eigenstates and magnetic response of spin-1 and spin-2 Bose-Einstein condensates. Phys. Rev. Lett. 84, $1066(2000)$.

9. Li, Z. B., Yao, D. X. \& Bao, C. G. Spin-thermodynamics of ultra-cold spin-1 atoms. J. Low Temp. Phys. 180, 200-213 (2015).

10. Ciobanu, C. V., Yip, S. K. \& Ho, T. L. Phase diagrams of F=2 spinor Bose-Einstein condensates. Phys. Rev. A 61, 033607 (2000).

11. Ueda, M. \& Koashi, M. Theory of spin-2 Bose-Einstein condensates: Spin correlations, magnetic response, and excitation spectra. Phys. Rev. A 65, 063602 (2002).

12. Isacker, $\mathrm{P}$ Van \& Heinze, S. Bose-Einstein condensates of atoms with arbitrary spin. J. Phys. A: Math. Theor. 40, 14811 (2007).

13. Chacon, E., Moshinsky, M. \& Sharp, R. T. $U(5) \subseteq O(5) \subseteq O(3)$ and the exact solution for the problem of quadrupole vibrations of the nucleus. J. Math. Phys. 17, 668 (1976).

14. Gheorghe, A. \& Raduta, A. A. New results for the missing quantum numbers labelling the quadrupole and octupole boson basis. J. Phys. A: Math. Gen. 37, 10951 (2004).

15. Kawaguchi, Y. \& Ueda, M. Spinor Bose-Einstein condensates. Phys. Rep. 520, 253 (2012).

16. Stamper-Kurn, D. M. \& Ueda, M. Spinor Bose gases: Symmetries, magnetism, and quantum dynamics. Rev. Mod. Phys. 85, 1191 (2013).

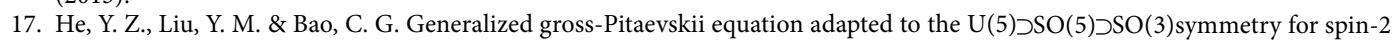
condensates. Phys. Rev. A 91, 033620 (2015).

18. Santos, L. \& Pfau, T. Spin-3 chromium Bose-Einstein condensates. Phys. Rev. Lett. 96, 190404 (2006). 
19. Makela, H. \& Suominen, K.-A. Ground states of spin-3 Bose-Einstein condensates for conserved magnetization. Phys. Rev. A 75, 033610 (2007).

20. Uchino, S., Otsuka, T. \& Ueda, M. Dynamical symmetry in spinor Bose-Einstein condensates. Phys. Rev. A 78, 023609 (2008).

21. Huhtamaki, J. A. M., Takahashi, M., Simula, T. P., Mizushima, T. \& Machida, K. Spin textures in condensates with large dipole moments. Phys. Rev. A 81, 063623 (2010).

22. Pasquiou, B., Marechal, E., Vernac, L., Gorceix, O. \& Laburthe-Tolra, B. Thermodynamics of a Bose-Einstein condensate with free magnetization. Phys. Rev. Lett. 108, 045307 (2012).

23. Situ, S. P. \& He, Y. Z. The spin evolution of spin- $3^{52} \mathrm{Cr}$ Bose Einstein condensate. Chin. Phys. B 20, 010310 (2011).

24. He, Y. Z., Chen, Z. F., Li, Z. B. \& Bao, C. G. Evaluation of the 52Cr-52Cr interaction via spin-flip scattering. Few-Body Syst. 46, 257 (2009).

\section{Acknowledgements}

Supported by the National Natural Science Foundation of China under Grants Nos. 11372122, 11274393, 11574404, and 11275279; the Open Project Program of State Key Laboratory of Theoretical Physics, Institute of Theoretical Physics, Chinese Academy of Sciences, China (No. Y4KF201CJ1); the National Basic Research Program of China (2013CB933601); and the Natural Science Foundation of Guangdong of China (2016A030313313).

\section{Author contributions}

Y.M.L. is for the theoretical calculation, Y.Z.H. is for the numerical calculation, C.G.B. propose the idea and write the paper.

\section{Competing interests}

The authors declare no competing interests.

\section{Additional information}

Supplementary Information is available for this paper at https://doi.org/10.1038/s41598-021-81133-7.

Correspondence and requests for materials should be addressed to C.G.B.

Reprints and permissions information is available at www.nature.com/reprints.

Publisher's note Springer Nature remains neutral with regard to jurisdictional claims in published maps and institutional affiliations.

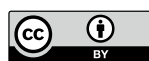

Open Access This article is licensed under a Creative Commons Attribution 4.0 International License, which permits use, sharing, adaptation, distribution and reproduction in any medium or format, as long as you give appropriate credit to the original author(s) and the source, provide a link to the Creative Commons licence, and indicate if changes were made. The images or other third party material in this article are included in the article's Creative Commons licence, unless indicated otherwise in a credit line to the material. If material is not included in the article's Creative Commons licence and your intended use is not permitted by statutory regulation or exceeds the permitted use, you will need to obtain permission directly from the copyright holder. To view a copy of this licence, visit http://creativecommons.org/licenses/by/4.0/.

(C) The Author(s) 2021 